\title{
Periodicities in the Io plasma torus
}

\author{
Michael E. Brown \\ Lunar and Planetary Laboratory, University of Arizona, Tucson
}

\begin{abstract}
We present a 6-month baseline of spatially resolved measurements of the Io plasma torus intensity and perpendicular ion temperature which we use to determine the periodicities of the torus during this time. We find large anticorrelated variations in the intensity and ion temperature which are periodic with the Jovian rotation rate (System III). The intensity variations are found to be a simple manifestation of the temperature variations, though no explanation for the temperature variations is apparent. Periodogram analysis shows an additional intensity periodicity which rotates $2.91 \pm 0.06 \%$ more slowly than System III. This period is found only in the intensity and not in the ion temperature. We conclude from these observations that the torus has a sinusoidal ion temperature variation locked into the rotation of Jupiter and that superimposed on this is a long-lived density pattern which rotates $2.91 \%$ more slowly than Jupiter. Based on the spatial structure and physical properties, we rule out all currently proposed mechanisms for the creation of these periodicities within the torus.
\end{abstract}

\section{Introduction}

Periodic variations within the Io plasma torus can reveal important clues into the nature of the Io-torus interaction and into the workings and properties of the entire Jovian magnetosphere. Unfortunately, past reports of periodicities and variations are inconsistent and inconclusive, making interpretation of the results difficult. Most previous observations are hampered by either an insufficient observational baseline, a lack of spatial resolution within the torus, or both.

In order to accurately determine the periodicities within the torus, we embarked upon a 6 -month intensive study of the visible emission from $\mathrm{S}^{+}$ions within the torus using spatially resolved long-slit high-resolution spcctra. With these spectra, we simultaneously measure intensities and perpendicular ion temperatures at all points along the torus equator.

This large data set allows us to firmly determine the existence of several different torus periodicities, to finally make a consistent picture which can explain earlier observations, and to rule out all present theoretical models for the formation of the observed periodicities.

\section{Past Observations}

Since the discovery of the Io plasma torus in 1976 [Kupo et al., 1976], numerous reports of variations in the torus intensity have been made (see the review by Thomas [1993]) but only a few observations have con-

Copyright 1995 by the American Geophysical Union.

Paper number 95JA01988.

0148-0227/95/95JA-01988\$05.00 tinued long enough for long-lived periodicities to be discerned. The most important long-term measurements to date are those taken by the Voyager ultraviolet spectrograph (UVS) [Sandel and Broadfoot, 1982a], the long-slit visible spectroscopy of Morgan [1985], and the Fabry-Perot spectroscopy of Roesler et al. [1984] and of Woodward et al. [1994].

The Voyager UVS obtained spectral scans of the Io plasma torus for a total of 4 months with Voyagers 1 and 2. Each point within a scan consists of the spectrum of the torus emission, integrated along the entire height of the torus, between 500 and $1700 \AA$. While this region of the spectrum contains many torus emission lines, all published results consider only the brightest line at $685 \AA$, a blend of three $\mathrm{S}^{++}$and one $\mathrm{O}^{++}$multiplet.

Sandel and Broadfoot [1982a] initially described this data set and found no systematic variations with magnetic longitude (throughout this paper, magnetic longitude will refer to the Jovian System III (1965) longitude, and the terms magnetic longitude and System III will be used interchangeably). Sandel and Broadfoot [1982b] discovered an Io-correlated brightness variation and interpreted this as an electron temperature variation caused by the interaction of the torus with Io. Roesler et al. [1984] pointed out that while the torus brightness showed no correlation with magnetic longitude, it was well organized with a period about $3 \%$ longer than Jupiter's rotation period. Sandel and Dessler [1988] carefully measured the non-System III period and derived a value of $10.22 \pm 0.02$ hours, or $3.0 \pm 0.2 \%$ longer than System III, which they termed the System IV period.

Roesler et al. [1984] made a 3-week-long groundbased study of $\mathrm{S}^{++} 9531 \AA$ emission from the torus using a large aperture Fabry-Perot spectrograph. Like 
the UVS, they found no variation at System III but a strong modulation at a period about $3 \%$ longer. Analysis by Woodward et al. [1994] shows that this variation has a period of $10.20 \pm 0.06$ hours, or $2.8 \pm 0.6 \%$ longer than System III, consistent with the System IV period proposed by Sandel and Dessler [1988].

Morgan [1985] obtained low-resolution long-slit. torus spectra for 15 days over a period of 4 months. The spectra included emissions at 3726 and $3729 \AA$ from $\mathrm{O}^{+}$and at $4069,4076,6717$, and $6731 \AA$ from $S^{+}$, species not studied by Roesler et al. [1984] or by the UVS. Contrary to the previous measurements, the $\mathrm{S}^{+}$emission intensity was found to be well correlated with magnetic longitude, with the peak occurring near a System III longitude, $\lambda_{\text {III }}$, of $\lambda_{\text {III }}=170^{\circ}$. No such peak is obvious in the $\mathrm{O}^{+}$emission lines, though they are weaker and noisier, and a variation of the magnitude of the $\mathrm{S}^{+}$variations cannot be ruled out. No attempt was made to search these data for any Io-related or other non-System III periodicity.

The first study explicitly designed to search for torus periodicities was performed by Woodward et al. [1994] using another large aperture Fabry-Perot instrument and observing the same $\mathrm{S}^{+} 6731 \AA$ emission line observed by Morgan [1985]. In direct contrast to Morgan, they found neither System III nor System IV periodicity; instead, the data varied consistently with a period of $10.14 \pm 0.06$ hours, $2.2 \pm 0.6 \%$ longer than System III.

In addition to UV and visible emissions, spacecraft observations have found that radio emissions are also associated with the torus. The narrowband kilometric (nKOM) radiation discovered by Voyager [Kaiser and Desch, 1980] emanates from the outer regions of the torus and appears modulated at a period of $10.21 \pm$ 0.03 hours (the System IV period). In addition, Daigne and Leblanc [1986] showed that nKOM appearance was also systematic with System III. They pointed out that while the appearance of the emission was modulated by the specific System III and System IV periods, the individual source regions appeared to lag corotation by between 1 and $8 \%$. This picture of the individual sources has been dramatically confirmed by using the radio direction finding capabilities of the Ulysses spacecraft [Reiner et al., 1993]. At the time of Ulysses closest approach, six separate nKOM sources were observed between 7 and $10 R_{J}$ with corotation lags between 3 and $8 \%$.

The inconsistencies amongst different observations are difficult to reconcile. Particularly puzzling are the results of Morgan [1985] and of Woodward et al. [1994] where the same emission line shows different periodicities. In an attempt to understand the differences in the previous observations and to better determine the irue torus periodicities, we conducted a 6-month intensive study of $S^{+} 6731 \AA$ emission in the torus, obtaining the most extensive torus data set to date. We find that the emission has strong variations at both the System III period and at one $2.91 \pm 0.06 \%$ longer, precisely the System IV period, but that the observed System III intensity variation is likely a manifestation of a discovered
System III ion temperature variation. The System IV period is a true height-integrated density enhancement in the torus.

\section{Observations and Reduction}

Observations of the Io plasma torus were obtained using the $60-\mathrm{cm}$ coudé auxiliary telescope (CAT) feeding the Hamilton echelle spectrograph [Vogt, 1987] at Lick Observatory. A total of 222 high-quality spectra spanning 53 nights of observation from December 2, 1991, until June 1, 1992, were used for this analysis. The spectra are high-resolution $(\lambda / \Delta \lambda \sim 40000)$, very long slit (slit length $\sim 6$ arc minutes) covering the torus [SII] (forbidden $\mathrm{S}^{+}$) emission doublet at 6717 and 6731 $\AA$. For each 40-min CCD integration the 10 arc second wide spectral slit was aligned parallel to the Jovian centrifugal equator and centered on Jupiter. Emission from Jupiter was attenuated by covering the center of the slit with a neutral density filter blocking about $99 \%$ of the light. A typical spectrum is shown in Figure 1. Emission from the two [SII] lines is slanted by the doppler shift caused by the torus rotation; reflected solar continuum from Jupiter appears as a vertical band of emission in the center of the spectrum and is usel to define the center of Jupiter accurately and to calibrate the intensity of the torus emission. Spatial resolution on the spectra is limited by the pixel size of the CCD to about 2.5 arc seconds, or about $0.1 \mathrm{RJ}$.

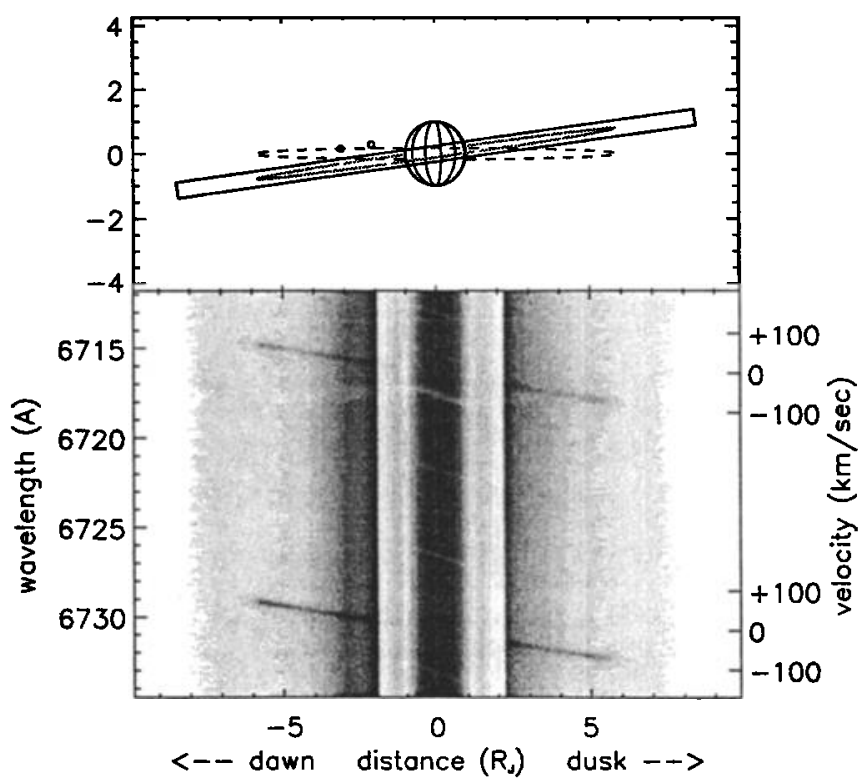

Figure 1. A long-slit spectrum of the Io plasma torus from the night of April 3, 1992. The upper panel shows the geometry of the Jovian system at the time of the observation with the dashed line indicating the orbit of Io and the solid line circling Jupiter tracing the path of the torus. The rectangle shows the projected size and orientation of the spectral slit. The bottom panel shows the spectrum. The two slanted emission lines are [SII] 6717 and $6731 \AA$ emission from the torus [from Brown, 1994a]. 
All of the spectra were reduced identically. First, each CCD frame was corrected using standard dark and bias subtracting and flat-fielding techniques. Wavelength scales were then obtained by comparison to spectra of a standard thorium-argon lamp, and the distance scale along the slit was determined by observations of double stars with known separations. The amount of Jovian scattered light (visible in Figure 1 as continuum emission extending past the radius of Jupiter) was determined from the spatial profile in spectral regions far from the [SII] emission. The scattered light was then removed by subtracting a synthetic spectrum consisting of the observed slit-integrated Jovian spectrum with the scattered light spatial profile.

The emission intensity at each radial distance was determined by summing the emission from the corrected, continuum-subtracted images in a $1.8 \AA$ region (corresponding to a width of $\pm 40 \mathrm{~km} \mathrm{~s}^{-1}$ ) centered on the expected line position. The torus ion temperature was measured by fitting a gaussian line profile to the ernission line at each radial distance and measuring the line width caused by the doppler motion of the ions. 'The temperature measured in this manner is the ternperature perpendicular to the magnetic field. The oiher component of ion temperature (parallel to the magnelic field) cannot be measured with these spectra. In the discussions below, "ion temperature" will refer to this measured perpendicular temperature unless otherwise noted. Absolute intensity of the torus emission was calibrated by comparison with the Jovian disk, which was assumed to have an intensity of $5.6 \mathrm{MR} \AA^{-1}$ [Woodman et al., 1979] (A Rayleigh is defined as $1 \mathrm{R}=\frac{1}{4 \pi} \times 10^{6}$ photons $\left.\mathrm{cm}^{-2} \mathrm{~s}^{-1} \mathrm{ster}^{-1}\right)$. Because these observations took place over a large range of Jupiter phase angles ( $t$ 10 degrees), the assumption of constant Jovian disk sur-

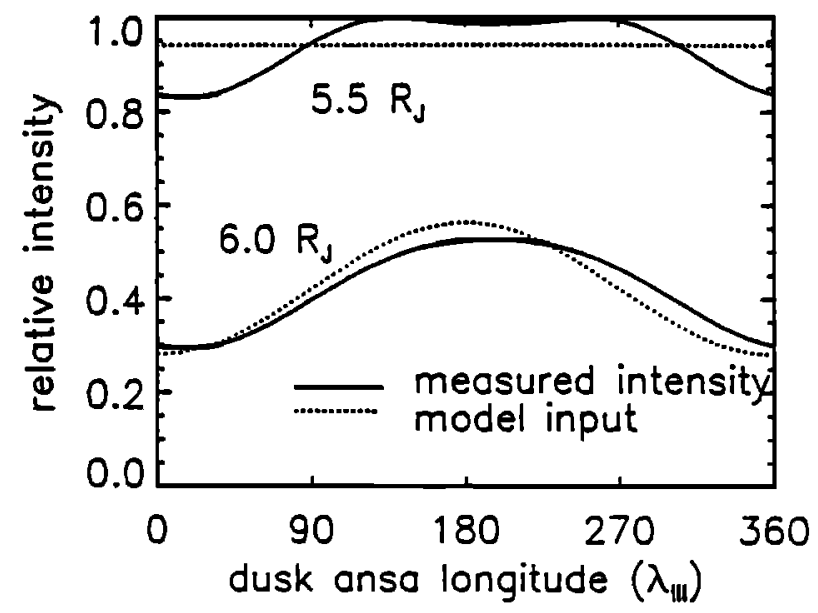

Figure 2. Measured intensities in a simulated torus versus the input intensities. Using the torus model of Bagenal [1994], a synthetic torus that is axially symmetric interior to $6 \mathbf{R}_{\mathbf{J}}$ and sinusoidally varying outside is constructed. Inside $6 \mathbf{R}_{\mathbf{J}}$, the apparent longitudinal structure is simply the result of projection effects, while outside, the measured intensity is close to the true intensity.

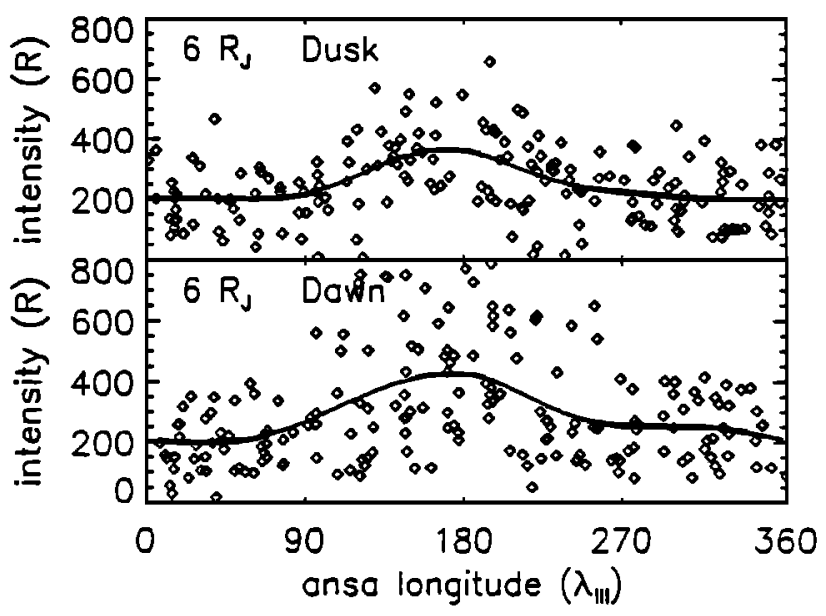

Figure 3. A plot of measured torus intensities at $6 \mathbf{R}_{\mathbf{J}}$ on the dawnside and duskside of the torus. The thick lines are $30^{\circ}$ sliding averages. On both sides of the torus, the torus intensity peaks at around $\lambda_{\text {III }}=170^{\circ}$. Much of the scatter around the averages is intrinsic to the torus; typical error measurements are $15 \%$.

face brightness is probably incorrect. This phase effect will introduce a small variation centered at opposition in the measured intensities but will in no way affect the analysis of much shorter timescale periodicities here.

In the analysis below, we consider only the data outside of $6 R_{\mathrm{J}}$, which is beyond the heavily studied ribbon region (see, for example, Schneider and Trauger, 1995). Inside of this distance, many of the apparent variations in the torus could be simply geometric jrojection effects. Beyond $6 \mathrm{R}_{\mathrm{J}}$, where the torus intensity is falling rapidly [see Brown, 1994b], projection effects are smaller. As an example, in Figure 2 we show simulations of the apparent variation in measured emission intensity of a torus that is axially symmetric inside of 6 $\mathrm{R}_{\mathrm{J}}$ and sinusoidally varying outside. (Model torus parameters are taken from Bagenal [1994]). Inside $6 \mathrm{R}_{\mathrm{J}}$, the measured emission is highly affected by the longitudinal asymmetry outside, and disentangling the true longitudinal intensity variations from those caused by geometric effects is a difficult task. Outside of $6 R_{\mathbf{J}}$ the torus geometry has little effect on the observations, and the measured intensity reproduces the true intensity.

\section{System III Periodicities}

In this section, we show that the torus has persistent anticorrelated System III variations in the [SII] $6731 \AA$ intensity and in the ion temperature. The intensity will be seen to be a manifestation of the temperature variation, but no explanation for the temperature variation is apparent.

\subsection{Intensities}

During the 6 months of observation, we could watch from night to night as a persistent bright spot seemed to rotate with the System III rate. This periodicity is illustrated in Figure 3, where we have plotted the in- 


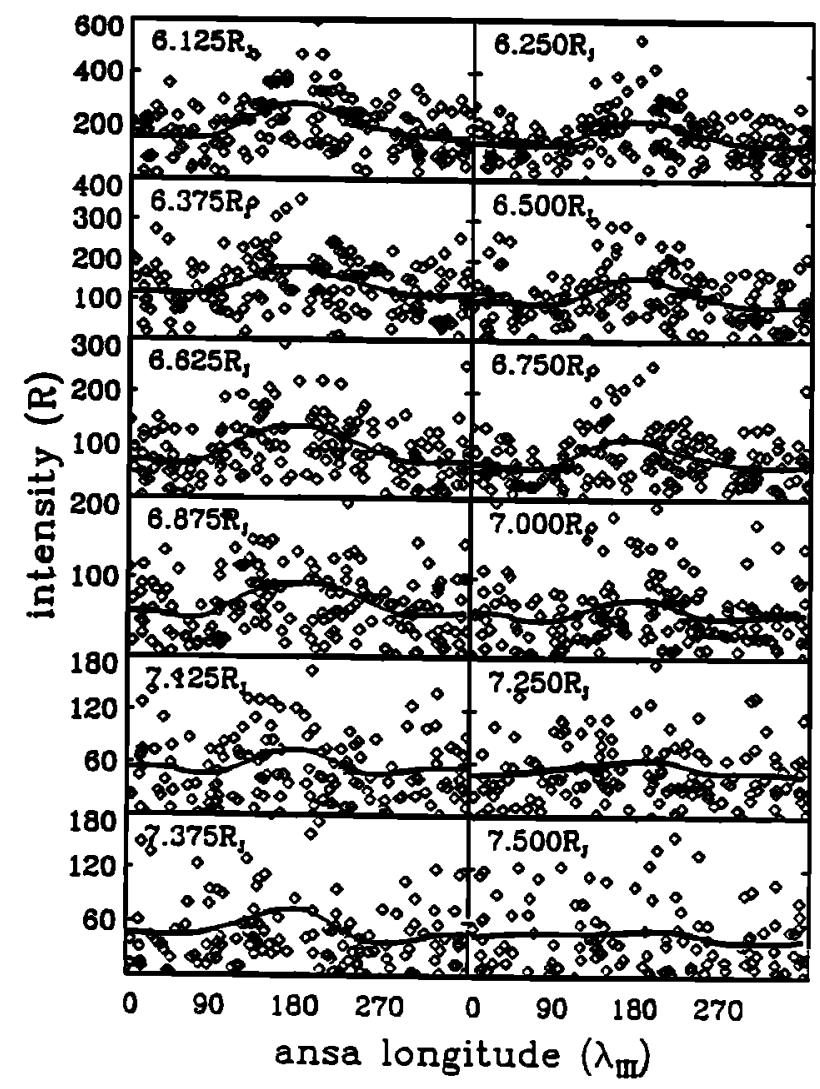

Figure 4. Torus intensity versus System III longitude for 12 different distances on the duskside of Jupiter. The thick lines are $30^{\circ}$ sliding averages. Note that System III modulation stays approximately constant in phase and magnitude until the signal disappears as the torus gets faint at its outer edge.

tensity as a function of System III longitude for points at $6 \mathrm{R}_{\mathbf{J}}$ on the dawnside and duskside of Jupiter. 'The thick lines show $30^{\circ}$ sliding averages of the data and confirm the general impression that the measured torus intensity is strongly correlated with magnetic longitıde. On both sides, the peak in emission occurs at approximately $\lambda_{\mathrm{III}}=170^{\circ}$, consistent with the results of MICrgan [1985]. Much of the scatter in these plots is inherent to the torus; estimated measurement errors are $\sim 15 \%$.

Figure 4 shows phase plots for all distances from 6 to $7.5 \mathrm{R}_{\mathrm{J}}$. At all distances, the torus intensity peaks around 180 degrees and has a structure similar to that shown in Figure 3. In order to quantify the location and size of the intensity peaks, we have fit the intensity variations to cosine curves with arbitrary phase, amplitude, and offset. Figure 5a shows the System III longitude at the peak of the fitted cosine curve at distances between 6 and $7.5 \mathrm{R}_{\mathrm{J}}$, and Figure $5 \mathrm{~b}$ shows the ratio between the cosine amplitude and the offset. On both the dawnside and duskside of Jupiter, the torus intensity peaks at about $\lambda_{\mathrm{III}}=180^{\circ}$ from $6 \mathrm{R}_{\mathrm{J}}$ outward as far as it can be measured. The relative amplitude of the intensity variation is also constant (or perhaps decreasing slightly) with distance.

We have attempted similar analysis using the ratio of the intensities of the 6716 and the $6731 \AA$ lines, which is a measure of the electron density. Unfortunately, we have found that the noise in this ratio is too large to extract meaningful results [see Brown 1994b], so we have no information about the behavior of the electron densities.

\subsection{Ion Temperatures}

The measurement of ion temperature in the torus is subject to greater error than that of the intensity (because of the difference between measuring the area and measuring the width of a Gaussian curve). To increase signal-to-noise for temperature measurements, we binned the data into 12 groups, each $30^{\circ}$ in longitude. We added together the spectra in each bin, and measured the ion temperature from these 12 binned spectra.

While the data are noisy, Figure 6 shows that a systematic variation with longitude clearly exists. On toth the dawnside and duskside, the ion temperature in the torus reaches a maximum at $\lambda_{\text {III }}=20^{\circ}$. The absolute magnitude of the variation is greater on the duskside where the average temperature is also greater, but the relative variation is the same on both sides. The variation can only be seen out to about $6.75 \mathrm{R}_{\mathrm{J}}$ before it disappears into the noise, but it is possible that like the intensity variation, this temperature variation extends to the observable limits of the torus.

A similar ion temperature variation has recently been found interior to $6 \mathrm{R}_{\mathrm{J}}$ [Schneider and Trauger, 1995]: Several nights of imaging data have shown large System III variations in the scale height of the ribbon feature, implying variations in the ion temperature parallel to the magnetic field (see (1) below). The phase of the parallel ion temperature variation is the same as that of the ion temperature variation measured bere,

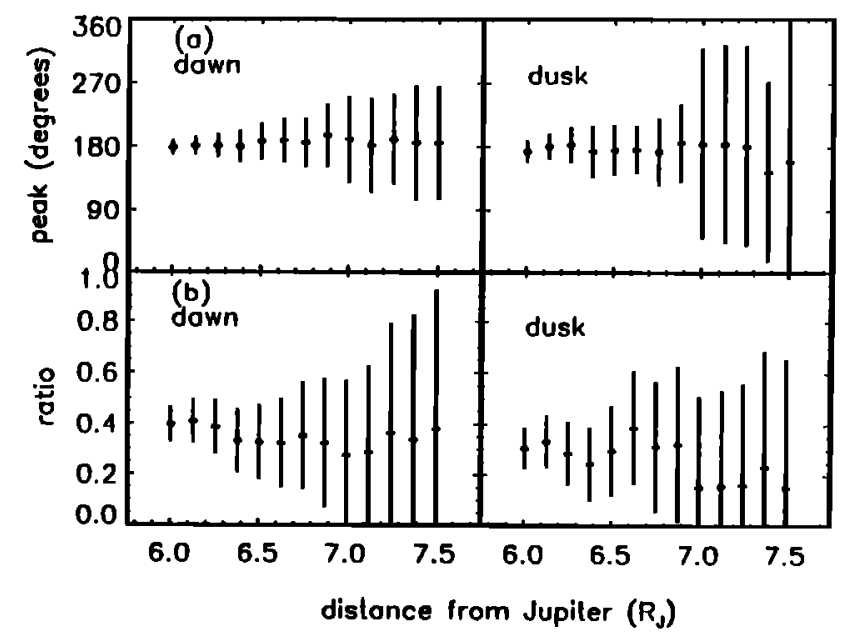

Figure 5. A sinusoidal curve plus a constant offset is fit to the phase plots at 13 distances on each side of Jupiter. (a) The peak point of the sinusoidal fit as a function of distance is shown. (b) The ratio of the amplitude of the sinusoidal modulation to the constant offset is shown. Note that both the phase and magnitude of the modulation do not change with distance. 


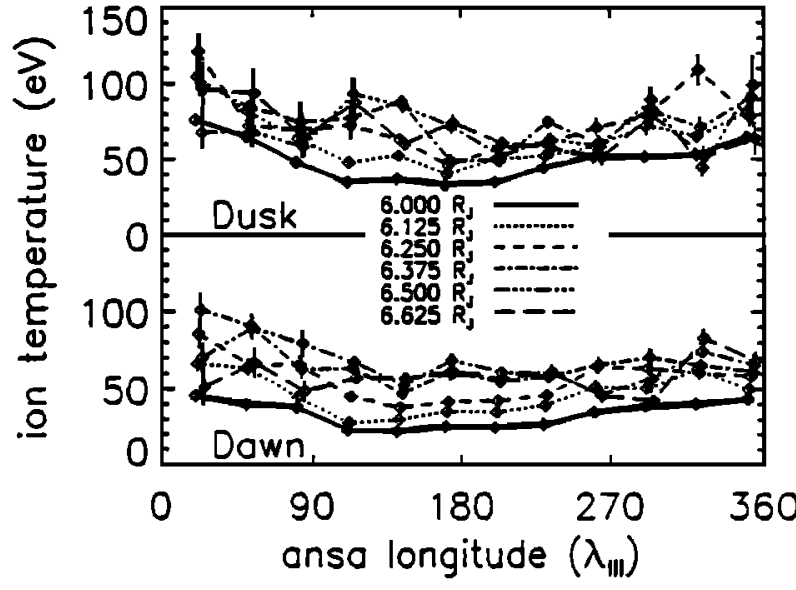

Figure 6. Perpendicular ion temperature measured from the line widths versus System III longitude for six distances on the duskside and dawnside of Jupiter. On both sides of the torus the temperature is strongly modulated and peaked near $\lambda_{\text {III }}=20^{\circ}$.

though the apparent amplitude of the parallel variation is much greater. Detailed comparison of these results must await removal of the geometry effects in the images and in our spectra interior to $6 R_{J}$.

\subsection{Implications}

4.3.1. Intensity variations caused by temperature variations. Torus intensities and ion temperatures are suggestively anticorrelated, as can be seen by comparing Figures 3 and 6 . It is possible that there is a direct causal link: a hotter torus has a greater scale height and a lower density, so an observer with a small slit looking near the torus equator will see a smaller fraction of a smaller total emission. When the torus is cold, the small scale height raises the density and brings a larger fraction of the larger emission within the small slit. We examine this possibility in detail below. As a word of caution, we note that the temperatures we have measured are those perpendicular to the magnetic field rather than the parallel temperatures which affect the scale heights. While the exact relation between perpendicular and parallel scale heights is unknown, we will assume that the temperatures have had time to isotropize and will be the same. The only available measurement, by Woodward [1992], suggests that the assumption of isotropy is reasonable.

The scale height of the torus, $H$, can be approximated by [Hill and Michel, 1976]

$$
H=\left(\frac{2 k T_{i}}{3 M \Omega^{2} \mathrm{R}_{\mathrm{J}}^{2}}\right)^{1 / 2},
$$

where $T_{i}$ is the ion temperature parallel to the magnetic field lines (not the measured perpendicular ternperature), $M$ is the effective ion mass [Bagenal and Sullivan, 1981], and $\Omega$ is the Jovian rotation rate. For $\mathrm{S}^{+}$, the scale height is

$$
H=0.12 \sqrt{T_{e V}} \mathbf{R}_{\mathrm{J}}
$$

where $T_{e v}$ is the ion temperature in electron volts. 'The fraction, $f$, of the ions which fall within the slit is given by

$$
f=\frac{\int_{0}^{S / 2} \exp \left(-z^{2} / H^{2}\right) d z}{\int_{0}^{\infty} \exp \left(-z^{2} / H^{2}\right) d z},
$$

where $S$ is the projected slit height in units of Jovian radii. This equation reduces to

$$
f=\operatorname{erf}(S / 2 H),
$$

where $\operatorname{erf}(x)$ is the error function of $x$. The emission intensity, $I$, is [Osterbrock, 1989] $I \sim n_{e} n_{i}$, where $n_{e}$ and $n_{i}$ are the electron and ion densities, respectively. Note that for the electron temperatures of interest, the intensity of the $6731 \AA$ [SII] emission is almost independent of electron temperature. Between 2 and $10 \mathrm{eV}$ the emission changes by less than $10 \%$.

Assuming that the electron scale heights are similarly affected and are equal to the ion scale heights at these distances [Bagenal, 1994], we can approximate

$$
I \sim \operatorname{erf}^{2}(S / 2 H) .
$$

This equation is only strictly valid if the ion and electron densities do not vary over the slit height, a condition approximately met by our small slit.

At $6 \mathrm{R}_{\mathrm{J}}$ on the duskside, the maximum measured temperature is $75 \mathrm{eV}$ and the minimum is $35 \mathrm{eV}$. For a slit height of $0.49 \mathrm{R}_{\mathrm{J}}$ (the average projected slit height during the observations), the fraction of ions and of electrons within the slit is 0.26 for the maximum temperature and 0.37 for the minimum temperature. The ratio between the intensity at the minimum and the maximum temperatures should be $(0.37 / 0.26)^{2}=2.0$. The measured ratio is approximately 1.8 . This close agreement suggests that the temperature variation alone could be the cause of the observed intensity variation.

In Figure 7 we show a comparison between the measured intensities and the intensities predicted from the above calculations at 6 and $6.25 \mathbf{R}_{\mathbf{J}}$ on the duskside. The observed intensities are well predicted from the measured temperatures, implying that the observed intensity variations are indeed a simple manifestation of temperature variations.

This new understanding of System III intensity variations provides a natural explanation for some of the discrepancies in past observations of the torus. All of the reports of large System III variations have been made with either long-slit spectroscopy centered on the centrifugal equator [Morgan, 1985] or from measurements made along the centrifugal equator in images [Schneider and Trauger, 1995] where the intensity is controlled by the scale height. Large-aperture observations of the torus (visible Fabry-Perot spectroscopy [Woodward et al. 1994, Oliverson et al., 1991; Roesler et al., 1984] and Voyager UVS spectroscopy [Sandel and Broadfoot, 1982a]) have always shown only very small or no variation with System III. Such a large aperture integrates emission from the entire torus, so the small-slit approximation of (5) is no longer valid. Instead, we can ap- 


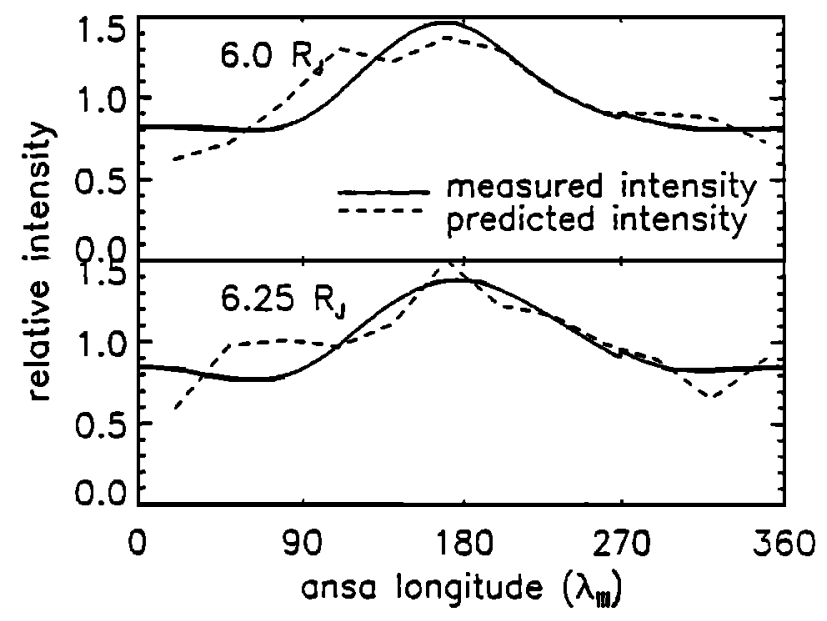

Figure 7. Torus intensity versus intensity variation predicted from the measured ion temperature. 'The measured and predicted intensities are well matched, showing that the observed System III intensity variation is simply a manifestation of the observed System III ion temperature variation.

proximate

$$
\begin{aligned}
I & \propto \int_{-\infty}^{+\infty} d z n_{e}(z) n_{i}(z) \\
& \propto \int_{-\infty}^{+\infty} d z \exp \left(-2 z^{2} / H^{2}\right) \\
& \propto 1 / H
\end{aligned}
$$

so, for the example cited above where the ion temperature varies between 35 and $75 \mathrm{eV}$, the intensity variation in a large aperture should be a factor of 1.46 . Woodward et al. [1994] show that their large aperture data show a System III modulation of about a factor of 1.44 , consistent with the above calculation. The even smaller System III variation in the UVS data [Sandel and Dessler, 1988] could be due to the stronger dependence of the UV emissions on electron temperature if the electron temperature shows periodicities similar to the ion temperature: lower temperature regions emit less per density but have smaller scale heights and correspondingly higher densities, so the effects can largely cancel.

This explanation for the intensity variations does not answer the question of what causes the variations; it merely changes it. A cause of temperature variations must now be found rather than intensity variations.

4.3.2. Magnetic anomaly. These observations are not explainable by the current version of the magnetic anomaly model, the only theory put forth thus far to explain persistent System III variations in the torus [Hill et al., 1983]. The general magnetic anomaly model predicts that torus properties can be affected by the surface magnetic field deviations on Jupiter (magnetic anomalies). The currently proposed mechanism by which the magnetic anomaly affects the torus is backsplash of energetic particles from auroral precipitation. With this mechanism, the torus density maximum should occur at the same location as the auroral maximum. We now know, however, that the intensity maximum at $180^{\circ}$ is not due to a density maximum but is simply a manifestation of a temperature and scale height variation. With this new understanding of torus periodicities we must also seek a new mechanism by which a magnetic anomaly might cause them.

4.3.3. Ion source variation. Most previous attempts to explain periodicities in the torus have shared the characteristic that the mechanism depends in some way on modulating the ion source rate to produce the torus variations. This mechanism is inconsistent with the observed spatial structure of the System III variations. If the intensity variation were caused by variations in the ionization rate, then the modulation would be greatest at the regions where the ionization occurs, and the modulation would rapidly disappear outside this region as the corotation lag in the torus smeared out the intensity peak during outward plasma transport. Brown [1994a] has shown that ionization in the torus occurs predominantly between 5.5 and $6.5 \mathrm{R}_{\mathrm{J}}$ and is strongly peaked at Io's orbital distance of $5.9 \mathrm{R}_{\mathbf{J}}$. Between 6 and $7 R_{J}$ the torus lags corotation by an average of about $2 \%$. Thus for the peak in the torus intensity to stay within about $20^{\circ}$ (the maximum allowed by the observations) throughout this range, the radial trinsport time would have to be slightly longer than 1 day, almost 2 orders of magnitude faster than estimated 80 day diffusion times [Strobel, 1989].

\section{System IV Period}

Periodicities approximately $3 \%$ longer than System III have been reported in past UV and the optical data. In this section, we show that a similar $2.91 \pm 0.06 \%$ longer period is visible in this data set, and we show that none of the current theories for generation of these periodicities adequately explains the observations. In particular, we demonstrate that the most popular explanation for these periodicities, the subcorotation of the torus, cannot explain the non-System III periodicity observed here.

\subsection{Periodograms}

To search our data for additional periodicities, we resort to the construction of Lomb-Scargle periodograms [Lomb, 1976; Scargle, 1982; Horne and Baliunas, 1986] which were developed to discern periodicities in unevenly sampled data. A thorough discussion of the properties of these periodograms and their application to torus data is given by Woodward [1992], Woodward et al. [1994], and Yang et al. [1991]. Essentially, the periodogram calculates the chi-square value for a sinusoidal fit at each potential period or, equivalently, cornputes the power spectrum for the data. While the periodograms are the best available method for searching for periods, they are also heavily influenced by noise and 
the details of the data sampling, so careful consideration needs to be given to the meaning of the calculated periodograms.

Various methods have been developed to quantify the errors in periodograms and to calculate the reliability of any predicted period. However, all rely on an estimate of the average effective sampling frequency, and no agreement exists on how best to determine this effective frequency. To circumvent this difficulty, we have extended the "shuffling" technique described by Woodward [1992] and Woodward et al. [1994] to give an estimate of probability that the highest peak in the periodogram is not caused by random errors. We take the data and randomly assign each point to a different one of the times of observation and recompute the periodogram for this new synthetic data set. The synthetic data set preserves the sampling pattern and noise characteristics of the original data, so it should give an idea of the height of peaks in the periodogram from these causes. We repeat this procedure 1000 times for each periodogram computed and assign 1,2 , and $3 \sigma$ values to the top 68,95 , and $99.7 \%$ values, respectively. It should be remembered that this calculation of the probability of the veracity of a peak is valid only for the highest peak of the periodogram and cannot be used to interpret any other peaks visible.

The accuracy of the frequency determination is generally assumed to be such that the error is the amount that would cause a phase shift of $360^{\circ}$ (or sometimes

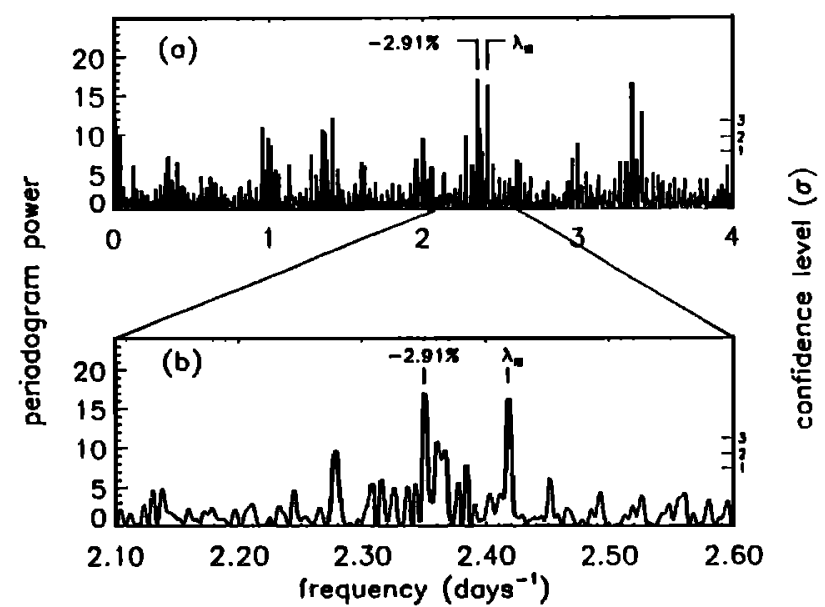

Figure 8. Periodograms of the intensity of the torus at $6 \mathrm{R}_{\mathrm{J}}$ on the dusk side over the 6 -month period of the observations. The periodogram power is plotted versus the frequency of all potential periods. The right axis shows the confidence level that the highest peak is caused by a real periodicity in the data as determined by the shuffle analysis. (a) The largest peaks occur near Jupiter's rotation frequency (2.4178 days $^{-1}$ ), and secondary peaks, related to the sampling times, occur at approximately 1 day $^{-1}$ and Jupiter's frequency plus and minus multiples of 1 day. (b) An expanded view of the region between 2.1 and 2.6 days -1 is shown. A peak at Jupiter's frequency appears, but the largest peak is at a frequency of 2.3503 days ${ }^{-1}, 2.91 \%$ longer than Jupiter's System III rotation. $180^{\circ}$ ) between the first and last sampling points of the data. While this method gives a useful order-ofmagnitude estimate for the accuracy, it does not lake into account either the sampling frequency or the uncertainties of the data. In order to more accurately assess the uncertainties in the frequency determination, we have constructed a large number of synthetic data sets sampled at the same times as the real data and having periodicities and uncertainties similar to the real data. The $3 \sigma$ accuracy of the frequency determination determined through this method is about $0.06 \%$, which corresponds to the amount of error that would cause the phase to change by $90^{\circ}$ between the beginning and end points of the sampling. While this is more accurate than these methods are generally assumed to be, this amount of accuracy appears to be correct: in the analysis below we find that the $3 \sigma$ scatter of the measurements of the System III frequency is actually less than $0.06 \%$ about the correct value.

\subsection{The Duskside}

Figure 8a shows the full periodogram for the data at $6 \mathrm{RJ}$ on the dusk side of the torus. The periodogram shows significant peaks at the System III rotation frequency ( 9.925 hours, or 2.418 days $^{-1}$ ) and at a period $2.91 \pm 0.06 \%$ slower (10.214 hours, or 2.350 days $^{-1}$ ), and smaller secondary peaks at frequencies precisely 1 and 2 days higher and lower. In addition there are peaks around frequencies of integer days. These secondary peaks are clearly caused by the details of the sampling (the average time between clumps of observations is 1 day or an integer multiple of a day) and are beat frequencies of the main torus periodicities. The only significant independent peaks are those around the System III period. Figure $8 \mathrm{~b}$ shows a portion of the periodogram centered on the System III rotation rate. A peak is visible at System III, as is expected from the analysis of this periodicity already above, but the highest peak in the periodogram occurs at a frequency of $2.350 \pm 0.01$ hours $^{-1}$, or a period of $10.214 \pm 0.006$ hours which is $2.91 \pm 0.06 \%$ longer than System III. In Figure 9 we show a phase plot of the data at the 10.214 hour period, and the periodicity is clearly evident. 'The magnitude of the modulation is slightly larger than the System III modulation in the same data.

The shuffle analysis shows that this peak is significant to much more than $3 \sigma$, but it cannot guarantee that the peak is not spurious and caused by the beating between a true System III period and another period or caused by the interaction between System III and the sampling times. We perform several tests to show that this possibility could not cause the $2.91 \%$ slower peak. First, we use the "pre-whitening" technique of Woodward et al. [1994]. The known System III variation (from Figure 3 ) is subtracted from the data, and the periodogram is recomputed and shown in Figure 10. The System III period is now clearly missing, and any spurious periodicity caused by the System III modulation, such as an interaction between the System III and Io orbital modulations, will be likewise missing. The 10.214-hour peak 


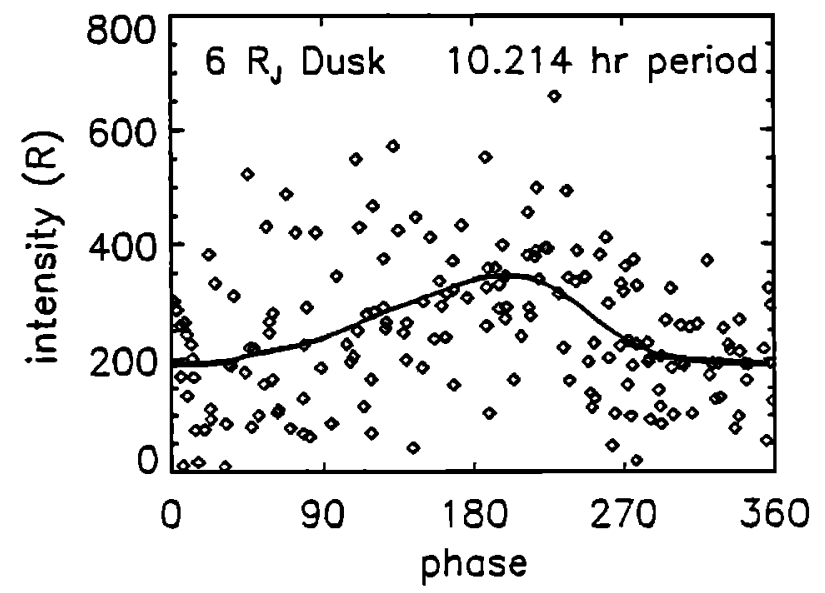

Figure 9. The torus intensity at $6 \mathrm{R}_{\mathrm{J}}$ on the duskside versus a 10.214 -hour period. The modulation at this period, $2.91 \%$ longer than System III, is obvious.

remains in precisely the same spot, showing that it is unrelated to System III. As an alternate approach, we also construct artificial data by sampling a purely periodic signal, consisting of the observed System III, at the times of our observations. The periodogram, shown in Figure $11 \mathrm{a}$, reveals a strong peak at the System III period but none elsewhere. Figure $11 \mathrm{~b}$ shows a periodogram computed from the same synthetic data but with artificial noise of the level observed added in. The System III peak is still completely dominant. These two tests show that the $2.91 \%$ longer period is related to neither the System III period, the Io orbital period, nor the sampling frequency.

Figure 12 shows periodograms for all distances from 5.875 to $6.750 \mathrm{R}_{\mathrm{J}}$ on the duskside. Both the System III and the $2.91 \%$ longer period are visible and unchanging until they begin to disappear into the noise as the torus gets faint. Phase plots for these distances are similar to the phase plot at $6 \mathrm{R}_{\mathrm{J}}$ above: the strength and phase of the modulation remain approximately constant, as was the case for the System III modulation.

\subsection{The Dawnside}

The dawnside of the torus also shows a strong periodicity of $2.91 \%$ longer than System III but only when data from a 22-day period of anomalous torus behavior are excluded. During this anomalous period, the torus was known to have undergone significant brightening and structural changes which greatly affected the dawnside out to about $6 \mathrm{R}_{\mathrm{J}}$ [see Brown, 1994b]. ('The duskside was also significantly affected but only interior to $6 \mathbf{R}_{\mathbf{J}}$ due to the dawn-dusk asymmetry of the torus.) Figure 13 shows a phase plot at a period of 10.214 hours for the dawn intensities at $6 \mathrm{R}_{\mathrm{J}}$ where we have plotted points from the 22 days between March 29 and April 20, 1992 as crosses and the remaining points as diamonds, with $30^{\circ}$ sliding averages shown separately for each. It is apparent that both the crosses and the diamonds are strongly modulated by the $2.91 \%$ longer period but that during the time of the dawn structural changes the phase of the modulation changed by about
100 degrees. A periodogram including all of the dawn data is contaminated by this phase shift; in an attempt to force the two separate phases to coincide, the periodogram constructs a spurious weak maximum at a period of 10.16 hours. Taking the normal and anomalous states separately, however, we find that both are consistent with the same System III and $2.91 \%$ slower periods found on the duskside, as can be seen in the periodograms plotted in Figure 14. For the normal state, the measured peak is at a value of $10.217 \pm 0.010$ hours, in good agreement with the 10.214 \pm 0.006 -hour period measured on the dusk side. Exterior to $6 \mathrm{R}_{\mathbf{J}}$, the measured phase change and periods are identical.

Reexamination of the dusk intensities shows the same phase shift. Inside of $6 \mathrm{RJ}_{\mathrm{J}}$ the phase shift is most apparent because the shift is accompanied by a dramatic brightening, similar to that seen on the dawnside at 6 $R_{J}$ shown in Figure 13. Outside of $6 R_{J}$ the phase shift is identical but not as apparent, because no brightening accompanied the shift. Recomputing the periodograms exterior to $6 R_{J}$ while excluding the 22 -day phase shift reveals no changes; the period of the non-System III modulation is still measured to be 10.214 hours. A more thorough analysis of this phase shift and brightening of the torus is currently underway.

\subsection{Temperature Variation}

Unlike the System III intensity variation, this intensity variation has no corresponding modulation in ion temperature, as can be seen by plotting the ion temperatures in a manner similar to Figure 6. The $2.91 \%$ longer period is not a simple scale height variation. Because the emission is insensitive to electron temperature in this temperature regime, the variation must be due to a true height-integrated density enhancement in the torus.

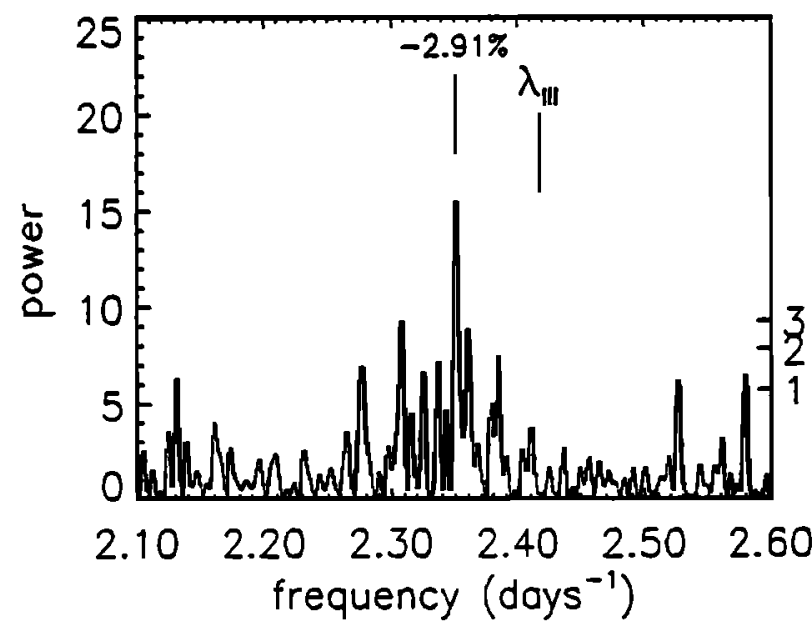

Figure 10. A periodogram computed after removing the System III signal using the "pre-whitening" technique of Woodward et al. [1994]. The right axis again shows the confidence level in the highest peak. 'The System III peak disappears, as expected, but the peak at 10.214 hours is not affected, showing that the nonSystem III period is not related to System III. 
Additional recent evidence for a System IV density enhancement comes from analysis of the hectometric emissions (HOM) observed by the Ulysses spacecraft [Kaiser et al., 1995]. The HOM was found to be modulated at a period about 3\% longer than System III, and the proposed source of the modulation is a System IV rotating density enhancement in the torus which blocks emission of the HOM from the auroral regions.

\subsection{Comparison With Other Observations}

The $10.214 \pm 0.006$-hour period reported here is similar to many previously reported measurements of nonSystem III torus periods. Analysis of the Voyager UVS data set [Sandel and Dessler, 1988] gives a value of 10.22 \pm 0.02 hours, and the Voyager nKOM data indicate a period of $10.21 \pm 0.03$ hours [Yang et al., 1991]. The ground-based measurements do not span as much time and so are more uncertain. A reanalysis of the Roesler et al. [1984] data [Woodward et al., 1994] finds a period of $10.20 \pm 0.06$ hours, while the data of Brown and Shemansky [1982] are too scattered to derive a meaningful independent period. These four data sets were combined by Sandel and Dessler [1988] to define a much more precise 10.224-hour period for System IV. With the new knowledge that the non-System III periodicity can change phase, we regard the combination of the data sets as unreliable and suggest that the most precise value from those data sets is the $10.22 \pm 0.02$-hour period from the UVS data.

The only other recent data in which a search for periodicities has been made are those of Woodward et al. [1994]. They determine a value for the non-System III periodicity of $10.14 \pm 0.06$ hours, where the stated uncertainty is 2 sigma. This result is marginally consistent with the values quoted above, though Woodward et al.

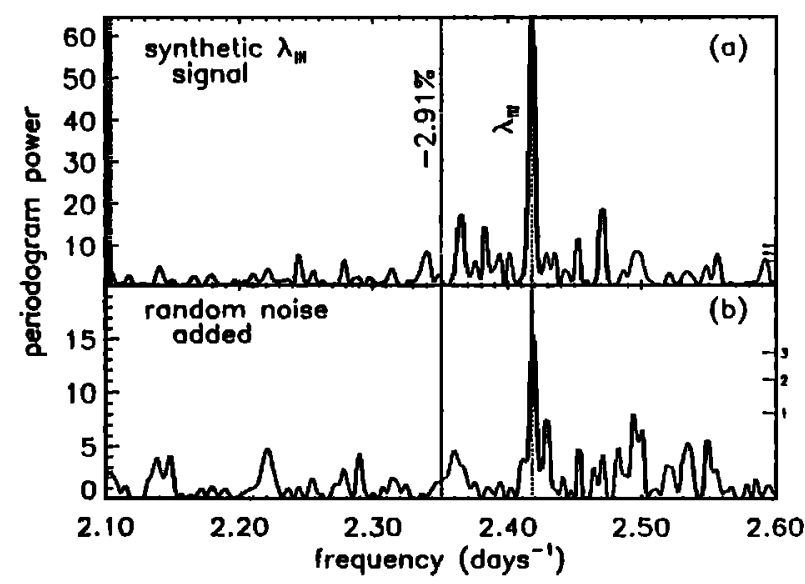

Figure 11. Periodograms computed for synthetic signals consisting only of the observed System III modulation and sampled at the same times as the real observations (a) with no noise added and (b) with noise of approximately the observed strength added. In both cases, only a System III signal appears. The unlabeled ticks on the right axis show the 1,2, and 3 sigma confidence levels for the highest peak. A purely System III signal sampled at these times cannot produce the non-System III period observed.

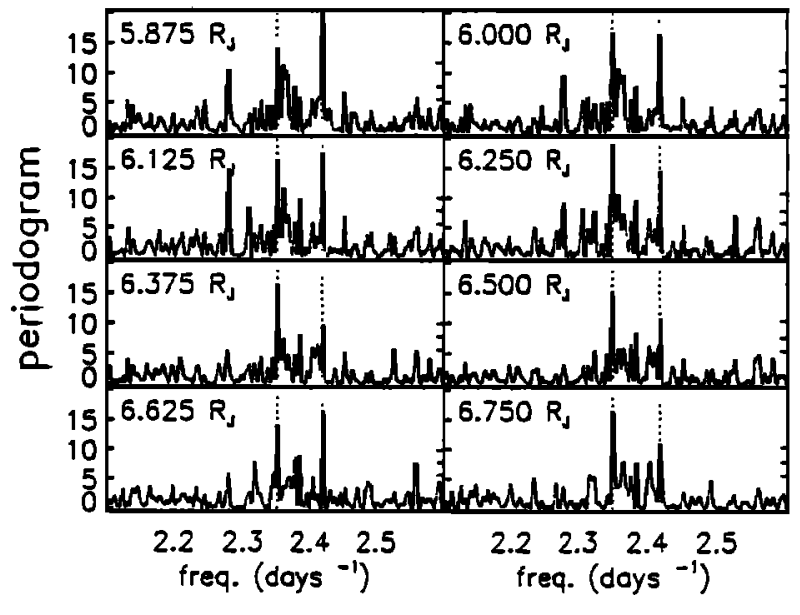

Figure 12. Periodograms computed at eight distances from Jupiter. The unlabeled tick marks on the right axes show the 1,2, and 3 sigma confidence levels in the highest peak. In all cases the System III and non-System III periods appear at exactly the same frequency.

show a phase plot using the proposed System IV 10.224hour phase where no modulation is apparent. Their period is similar to the 10.16-hour period derived from our dawn side periodogram when data from both the normal and anomalous torus states are combined. It is possible that during the observations of Woodward et al., the torus switched between two different phase states, destroying the integrity of the periodogram. A delailed examination of the phase plots could reveal if this possibility is true, though their data might not cover a long enough time period to clearly discern different states. R.C. Woodward (private communication, 1994) has recently verified that their data are consistent with a System IV period and a phase change in the middle of their observing run.)

Because of the agreement between all of these data sets, we conclude that Sandel and Dessler's [1988] hypothesis that the torus has a fundamental period approximately $2.91 \%$ longer than System III Jovian rotation rate is well supported, and we adopt their nomenclature and refer to this period as "System IV." Following Sandel and Dessler, we define the phase of the System IV period such that the emission peak of the torus occurs at $180^{\circ}$. (Note, however, that because of the observed month-long phase change, the definition of phase may be somewhat meaningless. We adopt the philosophy that the phase observed during the majority of the observation is the real phase, and the other phase was a temporary perturbation.) Thus the System III longitude of the central meridian of the System IV period, which we will denote by $\lambda_{\mathrm{IV}} ;(1992)$, is defined as

$$
\begin{aligned}
\lambda_{\mathrm{IV}}(1992)(t) & =\lambda_{\mathrm{III}}-24.6^{\circ} \times\left(t-t_{o}\right)+120^{\circ}, \\
& =845.90^{\circ} \times\left(t-t_{o}\right)+12^{\circ}
\end{aligned}
$$

where $t$ is in days and $t_{o}$ refers to Jan 1,1992 at 0000 UT (JD 248622.5). With the stated uncertainty, this 


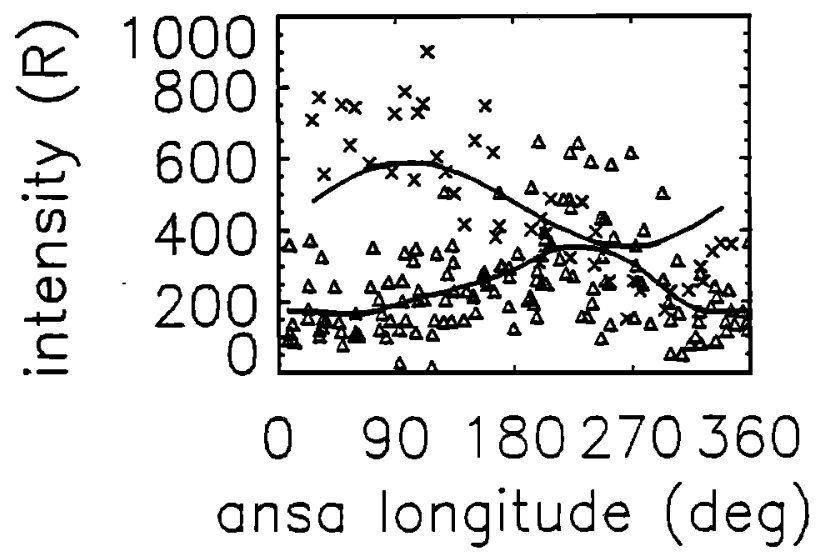

Figure 13. A phase plot of the intensity at $6 \mathrm{R}_{\mathrm{J}}$ on the dawnside versus the 10.214 hour period. Dates between March 29 and April 20 are marked as crosses, while others are shown as triangles. The regular 10.214-hour modulation is apparent for the triangles. For the month shown in crosses, a modulation at the same period exists, but the intensity has increased and the phase has changed by approximately 100 degrees.

longitude should be accurate to within 180 degrees for about 425 days before and after the observations, or October 1, 1990 until August 1, 1993.

\subsection{Comparison to Theory}

Four main types of theories have been put forth to explain the appearance of periodic behavior slower than Jupiter's rotation rate. We will show that the data shown here rule out all of these theories.

5.6.1. Corotation lag. The most commonly held belief, begun by Roesler et al. [1984] and continued by Thomas [1992] and recently by Woodward et al. [1994], is that the System IV periodicity is related to the corotation lag of the torus. The torus lags corotation by a few percent, so a long-lived structure in the torus will also lag corotation and appear periodic at a different period. Dessler [1985] and Sandel and Dessler [1988] argue convincingly that this explanation for a longer periodicity is theoretically implausible. We show now that in addition to being implausible, this idea is ruled out by the current observations.

The corotation lag in the torus varies with distance from Jupiter [Brown, 1994a]. If the $2.91 \%$ longer torus periodicity is caused by this corotation lag, then the period should change with distance from Jupiter as the rotation velocity changes. On the dusk side the torus lag ranges from $4-5 \%$ at $6 R_{\mathbf{J}}$ to close to $0 \%$ at 7.5 $\mathbf{R}_{\mathbf{J}}$. In this entire range of distances, the System IV period remains precisely fixed. Figure 15 shows a plot of the periodogram peaks for the System III and System IV periods versus distance from Jupiter. Superimposed on these points is the period that would be expected from the measured corotation lag [from Brown, 1994a] at these distances. No relation exists. The System IV period is not simply related to the corotation lag of the torus.
5.6.2. Separate magnetic anomaly. Dessler [1985] has suggested that the System IV periodicity is due to a hypothetical high-latitude component. of Jupiter's magnetic field that rotates more slowly than the rest of the planet. In this theory, the slowly rotating component has a magnetic anomaly similar to that of the System III rotating field, and this causes a bright spot in the same manner as the System III bright spot.

The current magnetic anomaly mechanism appears to fail to explain the System IV variations for one of the same reasons that it fails the System III variations. If the System IV periodicity is related to a variation in the ion source at this period, the intensity modulation would smear and disappear as the ions were transported outward through regions of corotation lag. The location of the peak of the System IV modulation is constant with distance, however, ruling out any such mechanism.

More generally, the System IV modulation is quite different than that of System III. While System III seems to be mainly periodic in ion temperature, with the emission intensity variation being a side effect, the System IV variation shows no such temperature effect and must be a true density increase along a magnetic field line. Thus no explanation can be made that the System IV variation is the same as System III, but just at a different period. The two variations are fundamentally different in nature.

5.6.3. Sampling. Another commonly held belief is that the appearance of the System IV period in the periodograms is spurious and simply caused by the details of the observational sampling. As noted by Thomas [1992], a spurious period of 10.21 hours can exist due to sampling modulated by Jupiter's rotation period and Io's orbital period.

Yang et al. [1991] and Woodward et al. [1994] go to great lengths to rule out this possibility by showing

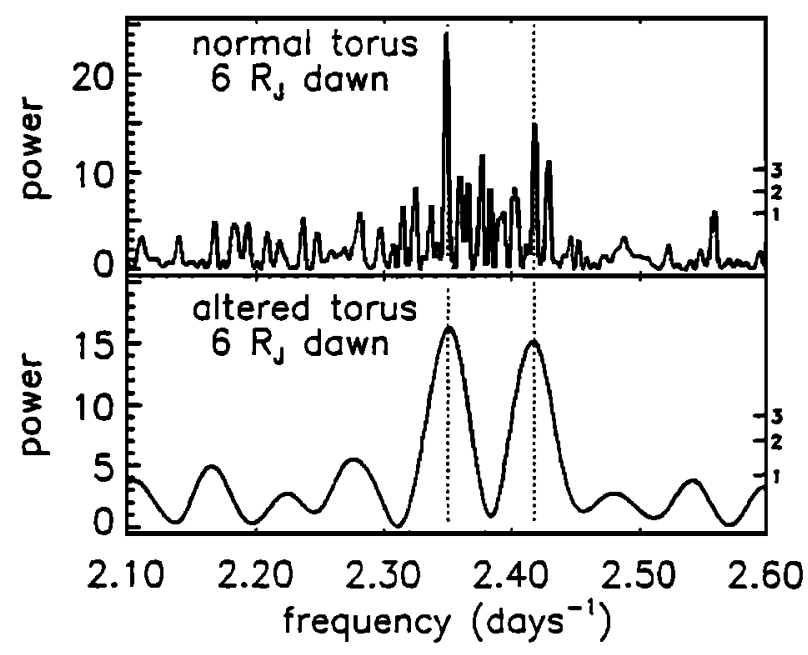

Figure 14. Periodograms of the dawnside showing the normal torus, which excludes the dates between March 29 and April 20, 1992, and the anomalous torus, between those dates, separately. In both cases, periods at the 9.925-hour System III and the 10.214-hour nonSystem III periods are visible. 
that if one subtracts out the System III variations from the data, the System IV remains. We have shown a similar result above and note that a periodicity such as this which goes through a sudden but reversible phase change can in no way be caused by details of the clata sampling.

5.6.4. Vortex. Horton and Smith [1988] proposed that the long-lived sub-System III periodicity was actually the manifestation of a solitary vortex in the torus powered by the torus velocity shear. The vortex would create a density enhancement that would rotate at a velocity intermediate between the ion velocity in the inner and outer edges of the torus. Details of this theory have not been worked out well enough to make specific predictions, but Sandel and Dessler [1988] make the point that while these vortices might occur, it is dificult to imagine them staying at a fixed rotation rate for 6 months or perhaps even 15 years.

In addition, these data show that one major signature of vortex activity is absent. To exist, a torus vortex must continuously circulate material from the outer reaches of the torus to the inner torus on a rapid timescale (Horton and Smith [1988] estimate the circulation time to be of the order of 1 hour). This circulation will cause the vortex to appear anomalously hot in the inner torus and cold in the outer torus. Neither of these effects are observed, suggesting that no such circulation is taking place.

\section{7. nKOM}

The emission of nKOM radiation is still difficult to explain, even with this new understanding of the torus. The hard question to answer is how can individual sources that rotate with corotation lags between 1 and $8 \%$ conspire to make an emission which appears periodic in both System III and a fixed period $2.91 \%$ longer? We propose that the appearance of the nKOM emission is intimately related to the periodicities discussed above, but that the individual sources rotate with the bulk velocity of the torus at their location. This situation is possible if nKOM sources are present in the outer torus and are moving with the bulk torus rotation appropriate for their distance, but their emission intensities are enhanced whenever they are either at $\lambda_{\text {III }}=40^{\circ}$ (the peak found by Sandel and Dessler [1988]), where the torus ion temperature is close to a maximum, or at the region of high density in the torus that rotates $2.91 \%$ slower (note that since the location of the nKOM peak relative to the torus density peak is not clear, it is possible that the nKOM emission is not enhanced at the peak density but rather somewhere else within the density phase). Thus, given a threshold emission intensity above which they have to radiate to be detected by the spacecraft, the nKOM emission sources will predominantly appear when enhanced at these two prefer red positions. When no sources are at these positions, little emission will be seen except for extraordinarily strong sources. As the spacecraft gets closer to the planet and the detection threshold drops, more and more emission

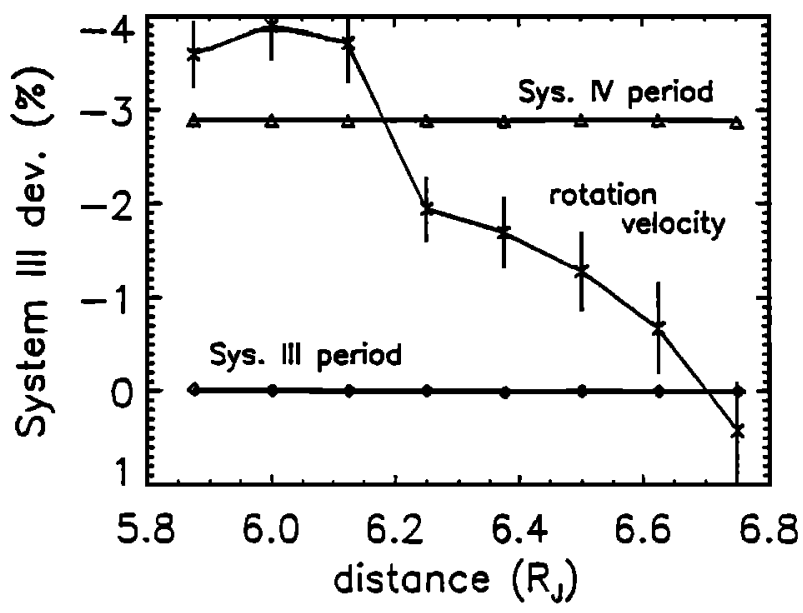

Figure 15. The measured peaks for the System III and the non-System III periods at eight distances on the dusk side of the torus. Superimposed on this plot are the measured corotation lags at these same distances [from Brown [1994a]. If the non-System III periodicity were simply related to the corotation lag, they would vary in the same fashion.

will be seen in the regions not related to the two preferred positions.

This explanation qualitatively explains the appearance, disappearance, and periodicities discussed by Kaiser and Desch [1980] and Daigne and Leblanc [1986] and the detailed structure observed by Reiner et al. [1993], but it rests on an untested assumption of low the torus temperatures and densities affect the nKOM emission. Unfortunately, there is no general agreement on the physical mechanism for the formation of the nKOM emission, though theories have been put forth [e.g., Jones, 1987; Fung and Papadopoulos, 1987].

\section{Discussion}

These observations allow a new consistent view of the Io plasma torus. The torus has ion temperature variations that are locked to the rotation of Jupiter. The temperature structure and its resulting intensity structure are constant with distance from Jupiter. Superimposed upon this structure is a long-lived density pattern that rotates $2.91 \%$ slower than Jupiter. The structure of this density pattern is also constant with distance from Jupiter. The System III and System IV periodicities are caused by the superposition of two pattern speeds. Individual ions and electrons move at a subcorotation velocity determined by the mass loading rate and unrelated to either the System III or System IV periods, in much the same way that the pattern speed of a spiral density wave in a spiral galaxy differs from the velocity of the underlying stars.

What can cause this dual periodic behavior in the torus? A System III temperature variation is potentially not difficult to understand. It can fit into the general framework of System III magnetospheric variabilities [see Hill et al., 1983] that might be caused by some 
sort of interaction between the Jovian surface magnetic field and the torus and magnetosphere. While the magnetic anomaly model in its present form cannot explain these variations, some variant might be a natural explanation for any System III effects in the torus.

The System IV period, however, is much more difficult to understand even just in principle. Unlike System III, it has no obvious planetary counterpart as an organizing factor. In addition, any conceivable mechanism which involves some interaction between Jupiter's rotation and any other regularly periodic occurrence, the orbit of Io, for example, has difficulty explaining the phase shift observed.

\section{Summary}

Using data from 6-months of high-resolution long-slit spectra of $\mathrm{S}^{+}$emission in the Io plasma torus, we have studied multiple torus periodicities. The main results are: (1) the observed emission intensity along the torus centrifugal equator is periodic in System III and brightest at about $\lambda_{\text {III }}=180^{\circ} ;(2)$ the ion temperature is also periodic with System III and is highest at $\lambda_{\text {III }}=20^{\circ}$ and lowest at $\lambda_{\text {III }}=170^{\circ}$; (3) the System III brightness modulation is likely a consequence of an ion temperature and scale height modulation; (4) because of the observed radial structure, the current version of the magnetic anomaly model cannot explain these intensity or temperature variations; (5) an intensity modulation at a period of $10.214 \pm 0.006$ hours, or $2.91 \pm 0.06 \%$ longer than System III, consistent with the proposed System IV period of Sandel and Dessler [1988], appears in the data, but no ion temperature variation at that period exists; (6) the System IV period underwent a sudden but reversible phase changes for approximately one month; and (7) the System IV period cannot be explained by the torus corotation lag, a vortex, or additional magnetic anomaly, nor is it a spurious period related to the sampling of the data.

Acknowledgments. I would like to thank Lick Observatory for the generous grant of substantial observing time. The Lick staff, and Tony Misch in particular, provided crucial assistance during the course of these observations. Many people have helped shape the course of this research, including Fran Bagenal, Alex Dessler, Mike Kaiser, Melissa McGrath, Imke de Pater, Frank Scherb, Nick Schneider, Bill Smyth, and Hy Spinrad. This research was supported, in various stages, by a grant from Calspace, by a NASA graduate fellowship, and by NASA through grant number HF-1056.01-94A from the Space Telescope Science Institute, which is operated by the Association of Universities for Research in Astronomy, Inc., under NASA contract NAS526555.

The Editor thanks N.M. Schneider and another referee for their assistance in evaluating this paper.

\section{References}

Bagenal, F., Empirical model of the Io plasma torus, I, Voyager measurements, J. Geophys. Res., 99, 11,043-11,062, 1994.
Bagenal, F., and J.D. Sullivan, Direct plasma measurements in the Io torus and inner magnetosphere of Jupiter, $J$. Geophys. Res., 86, 8447-8466, 1981.

Brown, M.E., Observations of mass loading in the Io plasma torus, Geophys. Res. Lett., 21, 847-850, 1994a.

Brown, M.E., The structure and variability of the Io plasma torus, $\mathrm{Ph} . \mathrm{D}$. dissertation, Univ. of Calif. at Berkeley, 1994b.

Brown, R.A., and D.E. Shemansky, On the nature of the SII emission from Jupiter's hot plasma torus, Astrophys. J., 263, 433-442, 1982.

Daigne, G., and Y. Leblanc, Narrow-band Jovian kilometric radiation: Occurrence, polarization, and rotation period, J. Geophys. Res., 91, 7961-7969, 1986.

Dessler, A.J., Differential rotation of the magnetic fields of the gaseous planets, Geophys. Res. Lett., 12, 299-302, 1985.

Fung, S.F., and K. Papadopoulos, The emission of narrowband Jovian kilometric radiation, J. Geophys. Res., 92, 8579-8593, 1987.

Hill, T.W., and F.C. Michel, Heavy ions from the Galilean satellites and the centrifugal distortion of the Jovian magnetosphere, J. Geophys. Res., 81, 4561-4565, 1976.

Hill, T.W., A.J. Dessler, and C.K. Goertz, Magnetospheric models, in Physics of the Jovian magnetosphere, edited by A.J. Dessler, pp. 353-394, Cambridge Univ. Press, New York, 1983.

Horne, J.H., and S.L. Baliunas, A prescription for period analysis of unevenly sampled time series, Astrophys. J., 302, 757-763, 1986.

Horton, W., and R.A. Smith, Solitary vorticies in the Io plasma torus, J. Geophys. Res., 93, 12,761-12,770, 1988.

Jones, D., Io plasma torus and the source of the Jovian narrow- band kilometric radiation, Nature, 327, 492-495, 1987.

Kaiser, M.L. and M.D. Desch, Narrow-band Jovian kilometric radiation: A new radio component, Geophys. Res. Lett., 7, 389-392, 1980.

Kaiser, M.L., M.D. Desch, and M.E. Brown, Evidence for an Io plasma torus influence on high-latitude Jovian radio emission, J. Geophys. Res., in press, 1995.

Kupo, I., Y. Mekler, and A. Eviatar, Detection of ionized sulfur in the Jovian magnetosphere, Astrophys. J., 205, L51-L53, 1976.

Lomb, N.R., Least-squares frequency analysis of unequally spaced data, Astrophys. Space Sci., 39, 477-485, 1976.

Morgan, J.S., Temporal and spatial variations in the Io torus, Icarus, 62, 389-414, 1985.

Oliverson, R.J., F. Scherb, and F.L. Roesler, The Io sulfur torus in 1981, Icarus, 93, 53-62, 1991.

Osterbrock, D.E., Astrophysics of Gaseous Nebulae and Active Galactic Nuclei, Univ. Sci. Books, Mill Valley, Calif., 1989.

Reiner, M.J., J. Fainberg, R.G. Stone, M.L. Kaiser, M.D. Desch, R. Manning, P. Zarka, and B.-M. Pederson, Source characteristics of Jovian narrow-band kilometric radio emissions, J. Geophys. Res., 98, 13,163-13,176, 1993.

Roesler, F.L., F. Scherb, and R.J. Oliverson, Periodic intensity variation in [SIII] $9531 \AA$ emission from the Jupiter plasma torus, Geophys. Res. Lett., 11, 128-130, 1984.

Sandel, B.R., and A.L. Broadfoot, Io's hot plasma torus A synoptic view from Voyager, J. Geophys. Res., 87, 212218, 1982a.

Sandel, B.R., and A.L. Broadfoot, Discovery of an Io-correlated energy source for Io's hot plasma torus, J. Geophys. Res., 87, 2231-2240, 1982b.

Sandel, B.R. and A.J. Dessler, Dual periodicity of the Jovian magnetosphere, J. Geophys. Res., 93, 5487-5504, 1988.

Scargle, J.D., Studies in astronomical time series analysis, 
II, Statistical aspects of spectral analysis of unevenly sampled data, Astrophys. J., 263, 835-853, 1982.

Schneider, N.M., and J.T. Trauger, The structure of the Io torus, Astrophys. J., in press, 1995.

Strobel, D.F., Energetics, luminosity, and spectroscopy of Io's torus, in Time Variable Phenomena in the Jovian System, edited by M.J.S. Belton, R.A. West, and J. Rahe, NASA Spec. Publ. SP-494, 1989.

Thomas, N., Optical observations of Io's neutral clouds and plasma torus, Surv. Geophys., 13, 91-164, 1992.

Thomas, N., The variability of the Io plasma torus, J. Geophys. Res., 98, 18,737-18,750, 1993.

Vogt, S., The Lick Observatory Hamilton echelle spectrometer,, Publ. Astron. Soc. Pac., 99, 1214-1228, 1987.

Woodman, J.H., W.D. Cochran, and D.B. Slavsky, Spatially resolved reflectivities of Jupiter during the 1976 opposition, Icarus, 97, 73-83, 1979.
Woodward, R.C., Sulfur emission and periodicity of the Jupiter plasma torus in 1988, Ph.D. dissertation, Univ. of Wisconsin, Madison, 1992.

Woodward, R.C., F. Scherb, F.L. Roesler, and R.J Oliverson, Periodic intensity variations in sulfur emissions from the Io plasma torus, Icarus, 111, 45-64, 1994.

Yang, Y.S., A.J. Dessler, and B.R. Sandel, Is System IV independent of System III?, J. Geophys. Res., 96, 38193824, 1991.

M.E. Brown, Lunar and Planetary Laboratory, University of Arizona, Tucson, AZ, 85721. (email: mbrown@lpl.arizona.edu)

(Received January 31, 1995; revised June 20, 1995; accepted June 23, 1995.) 